\title{
EVALUACIÓN DE LA VIDA ÚTIL DE UNA PASTA DE TOMATE MEDIANTE PRUEBAS ACELERADAS POR TEMPERATURA

\begin{abstract}
Resumen
El objetivo de esta investigación fue estimar la vida útil de una pasta de tomate mediante pruebas aceleradas, empleando la degradación de color como indicador de deterioro. El producto se almacenó a $40^{\circ} \mathrm{C}, 45^{\circ} \mathrm{C}$ y $50^{\circ} \mathrm{C}$ durante 110 días, 120 días y 42 días, respectivamente. Se realizaron como mínimo 6 muestreos para cada temperatura y los resultados obtenidos se utilizaron para definir la cinética de esta reacción de deterioro. La cinética de la reacción encontrada era de orden cero y las constantes cinéticas obtenidas fueron $0,031^{-1}, 0,064$ día $^{-1}$, y 0,097 día ${ }^{-1}$, en orden creciente de temperatura. Con estas velocidades de reacción y el modelo de Arrhenius se obtuvo además un valor de la energía de activación de $95680 \mathrm{~J} / \mathrm{mol}$. Por último, se obtuvo una relación para estimar la vida útil de la pasta de tomate, cuya ecuación general es Vida útil $=10(4,259-0,053 \bullet T)$.
\end{abstract}

Palabras clave: vida útil, pasta de tomate, cinética de deterioro, energía de activación.

\begin{abstract}
The purpose of this study was to estimate the shelf life of tomato paste using accelerated temperatures. The deterioration varable used was color. The product was stored at $40^{\circ} \mathrm{C}, 45^{\circ} \mathrm{C}$ and $50^{\circ} \mathrm{C}$ for 110,120 and 42 days. It was sampled a least 6 times for each temperature and the results were used to define the kinetics for this deterioration. The order obtained for the kinetics' reaction was zero and the constants found were 0,031 day $^{-1}, 0,064$ day ${ }^{-1}$, y 0,097 day $^{-1}$, in the same temperature order. Making use of the Arrhenius Model, the calculated activation energy was $95680 \mathrm{~J} / \mathrm{mol}$. Finally, the expression for estimating the tomato paste shelf life is described by the equation Shelf life $=10^{\left(4,259-0,053^{\bullet} T\right)}$.
\end{abstract}

Keywords: Shelf life, tomato paste, deterioration kinetic, activation energy.

Recibido: 09 de Febrero del 2010 • Aprobado: 31 de Marzo del 2011

\section{INTRODUCCIÓN}

En la industria de los alimentos se requiere conocer la vida útil de los productos para asegurar a los consumidores finales que el alimento adquirido mantiene todas sus características en buen estado.

La vida útil de un producto depende de muchos factores, como por ejemplo: ambientales, humedad, temperatura a la cual se expone, el proceso térmico que sufre, de la calidad de las materias primas que lo componen, entre otros. El resultado de la exposición a estos factores produce cambios en las cualidades del alimento que impide su comercialización como puede ser la pérdida de nutrientes, cambios de sabor, color o textura.

Casp y April (1999) indican que hay un determinado tiempo, después de haber elaborado el producto, en el que se mantienen sus propiedades sensoriales y de seguridad, bajo determinadas condiciones de almacenamiento.

El final de la vida útil de un producto se alcanza cuando ya no mantiene las cualidades requeridas para que el consumidor final lo utilice (Kuntz, 1991).

La industria de alimentos se interesa por conocer la vida útil de los productos elaborados para suplirlos al mercado sin problemas de seguridad o de rechazo por parte de los consumidores. 
Los estudios de vida útil acelerados permiten obtener información en tiempos relativamente cortos; consisten en incubar el alimento bajo condiciones controladas y a diferentes temperaturas. Estas temperaturas, en general, deben ser mayores a las de almacenamiento y comercialización para permitir que las reacciones de deterioro se aceleren y se obtenga una respuesta en un tiempo comparativamente corto respecto a otros métodos.

Para los estudios acelerados se debe establecer un diseño experimental que contemple las variables a evaluar y controlar las variables que no se desean evaluar para evitar que interfieran en las mediciones, y por ende, en los resultados (Labuza y Schmidt, 1985).

Las pruebas de laboratorio asemejan en lo posible las condiciones reales pero existen variables como las condiciones de transporte, cambios de presión, variaciones de temperatura, que son difíciles de duplicar. Por esto, los resultados obtenidos son estimaciones de la vida útil del alimento.

Debido a que no hay mayor información respecto a la vida útil de pastas de tomate y su energía de activación, este trabajo tiene como propósito determinarla mediante pruebas aceleradas por temperatura.

\subsection{Algunas formas de deterioro de los alimentos}

Cuando un alimento sufre un proceso de escaldado, salado, secado, congelación, etc., su estructura interna cambia provocando daños en las membranas. Por lo tanto, las paredes de la célula se rompen y las sustancias que antes estaban separadas, se ponen en contacto y reaccionan. Existe una gran cantidad de reacciones que se pueden dar, pero la mayoría pueden clasificarse dentro de las siguientes áreas:

- Oscurecimiento no enzimático. Una serie de reacciones complejas que inician con compuestos reductores y grupos amino que producen sabores amargos, pigmentos oscuros, pérdida de solubilidad de las proteínas y pérdida de características de sabor. Muchos alimentos contienen, en alguna proporción, ácidos de grasas insaturadas en la forma de triglicéridos. La reacción entre esta grasa y el oxígeno está descrita por un mecanismo de radicales libres complejo y produce compuestos volátiles de bajo peso molecular como aldehídos, cetonas, ácidos y alcoholes.

- Pérdida de vitaminas. Esto conlleva a la pérdida del valor nutricional del alimento. La destrucción de las vitaminas puede ocurrir a través de varios mecanismos como es la hidrólisis debido a la luz, calor o ácidos, a la oxidación directa en presencia de oxígeno y la participación de éstas en reacciones redox.

- Cambio de color. El color natural de los alimentos se pierde como consecuencia de varios tipos de reacciones, ya que el color se debe a compuestos solubles en agua o aceite. El color se pierde también por la oxidación directa de carotenoides o co-oxidación de lípidos.

- Actividad enzimática. Si los alimentos no se someten a tratamientos térmicos para inactivar las enzimas, éstas pueden catalizar ciertas reacciones que producen sabores, colores o texturas indeseadas (Labuza, 1984).

\subsection{Medición de deterioro del color}

El color de los alimentos es, generalmente, un indicador de madurez o descomposición.

$\mathrm{Si}$ el alimento es un líquido como el vino o jugo de uva, o si se puede obtener de él un extracto de color, es posible emplear varios tipos de colorímetros o espectrofotómetros para medir su color. En estos instrumentos se dirige a través del producto un haz de luz de una longitud de onda seleccionada. El grado de absorción de la luz dependerá del color del líquido y su intensidad. Si uno de los líquidos es un jugo, y el otro es un jugo diluido con agua, la última muestra transmitirá una mayor fracción de luz que ingresa (Potter, 1978).

Si el alimento es un líquido o un sólido, se puede medir su color al compararlo con los colores definidos de placas o discos patrón. Estos existen en gran variedad de tonos, con ellos el color se puede medir en forma mucho más precisa; la luz reflejada por un objeto de color puede ser dividida en tres componentes a los que 
se les denomina valor, matiz y croma. El valor se refiere a la claridad u oscuridad del color. El matiz se refiere a la longitud de onda predominante que determina si el color es rojo, verde o amarillo. El croma se refiere a la intensidad del color. Equipos como el Medidor de Color y Diferencia de Color del Hunter Lab miden estos valores cromáticos en la superficie de un alimento (Potter,1978).

\subsection{Modelo para la degradación cinética}

Una de las particularidades de los cambios en los atributos de los alimentos, como el color, textura, sabor, es que estos responden a modelos cinéticos de orden cero o de primer orden (Díaz,2004; Potter, 1978; Labuza \& Schmidt,1985; Kuntz, 1991; Casp y April,1999; CITA,2005).

El modelo para una reacción de orden cero se presenta como:

$$
-\frac{d X}{d t}=k
$$

Cuando la ecuación (1) se integra y reacomoda, tiene la forma de una línea recta con pendiente $k$, que es la constante específica de reacción, cuyo valor depende de la temperatura y, $\mathrm{X}$ es el atributo.

$$
X_{f}=X_{0}-k t_{u}
$$

Con $X_{0}$ como la intersección con el eje Y.

\subsection{Dependencia de la temperatura}

El modelo de Arrhenius describe la relación de la constante de velocidad de reacción con la temperatura, esta dependencia se muestra en la ecuación (3):

$$
k=A e^{\left(-E_{a} / R T\right)}
$$

Al aplicar logaritmos a ambos lados de la ecuación (3) se obtiene la ecuación de una línea recta con pendiente $\mathrm{E} a / \mathrm{R}$, y se despeja el término $E_{a}$ para obtener el valor de la energía de activación:

$$
\ln k=\ln A-\frac{E_{a}}{R} \cdot \frac{1}{T}
$$

Donde:

$k \quad$ constante de velocidad de reacción

A factor de frecuencia

$E_{a}$ energía de activación

$R \quad$ constante de los gases ideales

$T$ temperatura absoluta (K).

\section{METODOLOGÍA Y EQUIPO EXPERIMENTAL}

\subsection{Producto}

Los componentes principales de este producto son pasta de tomate, agua y sal. Debido a la naturaleza del producto, el atributo que en mayor grado es afectado por condiciones de proceso y almacenamiento es el color.

\subsection{Empaque del producto}

El empaque es flexible. La estructura del empaque para los productos hechos a base de tomate, contiene poliéster, tintas, adhesivos, polietileno y aluminio.

El poliéster es una barrera contra la humedad, posee alta resistencia a la ruptura y a las altas temperaturas. Las tintas funcionan como barrera contra la luz. El adhesivo tiene la función de unir las capas de los demás materiales y si está en contacto con el alimento, en las uniones y en los bordes de los laminados, debe estar presente en la cantidad mínima. El polietileno es flexible, fácilmente estirable, es una barrera contra el vapor de agua, no tiene olor ni sabor y es fácilmente sellable por calor. El aluminio, mejor conocido como "foil" por su nombre en inglés es una lámina de barrera contra los gases y la luz. 


\subsection{Variable de respuesta}

Se seleccionó el color como la variable de respuesta debido a que este atributo es uno de los que sufre mayor cambio en este tipo de productos.

La determinación del color se realiza con un colorímetro siguiendo el procedimiento descrito en el manual de procedimiento del equipo. Se utiliza la escala L-a-b del método Hunter Lab para definir el color del tomate. Se calibra el equipo antes del inicio de cada medición con una placa de color blanca. Posteriormente, se coloca la muestra en una placa de petri de $60 \mathrm{~mm} \mathrm{x}$ $15 \mathrm{~mm}$ y se lee directamente los valores dados por el equipo; donde L indica la luminosidad y el grado de oscurecimiento, a indica la porción del color rojo al verde y b indica la porción del color amarillo al azul (Rondon, Pacheco \& Ortega, 2004; Melgaard,1999; Neter,1983). Las determinaciones se realizan por duplicado.

Debido a que la medición de color se puede representar como un punto ubicado en el espacio, es decir, con ejes L, a y b, y colocado en la sección positiva del mismo, se pueden calcular los vectores correspondientes a cada muestreo utilizando la ecuación:

$$
\text { Vector }=\sqrt{L^{2}+a^{2}+b^{2}}
$$

\subsection{Definición de la periodicidad de análisis}

En el Cuadro 1 se presentan los intervalos de tiempo de muestreos; el tiempo global más corto de 42 días corresponde a la mayor temperatura utilizada para el estudio. La definición de la periodicidad se estableció en pruebas previas.

\subsection{Metodología y procedimiento experimental}

Se toman muestras del mismo lote de producción para el estudio. Se almacenan en refrigeración a $5^{\circ} \mathrm{C}$ para mantenerlas "frescas" hasta su colección en la incubadora para iniciar el estudio de vida útil acelerado. Luego se colocan muestras en la incubadora a la temperatura seleccionada y se procede a la toma de muestras tal como quedó establecido en la definición de la periodicidad de análisis (García, 2008).

\subsection{Equipo experimental}

Incubadora: Lab Line Imperial III-30, que permite establecer las temperaturas de interés ( $40^{\circ} \mathrm{C}, 45^{\circ} \mathrm{C}$ y $\left.50^{\circ} \mathrm{C}\right) \pm 1^{\circ} \mathrm{C}$; cuenta con el espacio suficiente para colocar las muestras de pasta de tomate que deben tomarse periódicamente para el análisis del color de la pasta.

En el Cuadro 2, se presentan los equipos para la medición del color.

\section{DISCUSIÓN DE RESULTADOS}

Los resultados del cambio de color en función del tiempo para cada temperatura se muestran en la Figura 1, Figura 2 y Figura 3. La pendiente en cada una de ellas representa la constante de reacción o deterioro del color.

Cuadro 1. Secuencia de muestreo para el análisis de pasta de tomate.

\begin{tabular}{cccc}
\hline $\begin{array}{c}\text { Temp. almacen. } \\
\left({ }^{\circ} \mathrm{C}\right)\end{array}$ & $\begin{array}{c}\text { Período } \\
\text { medición } \\
(\mathrm{d})\end{array}$ & $\begin{array}{c}\text { Tiempo máximo } \\
\text { almacenamiento } \\
(\mathrm{d})\end{array}$ & $\begin{array}{c}\text { Muestreo } \\
(\mathrm{d})\end{array}$ \\
\hline 40 & $20 / 30$ & 110 & $20,40,60,80,110$ \\
45 & $30 / 15$ & 120 & $30,60,75,105,120$ \\
50 & 7 & 42 & $7,14,21,28,35,42$ \\
\hline
\end{tabular}




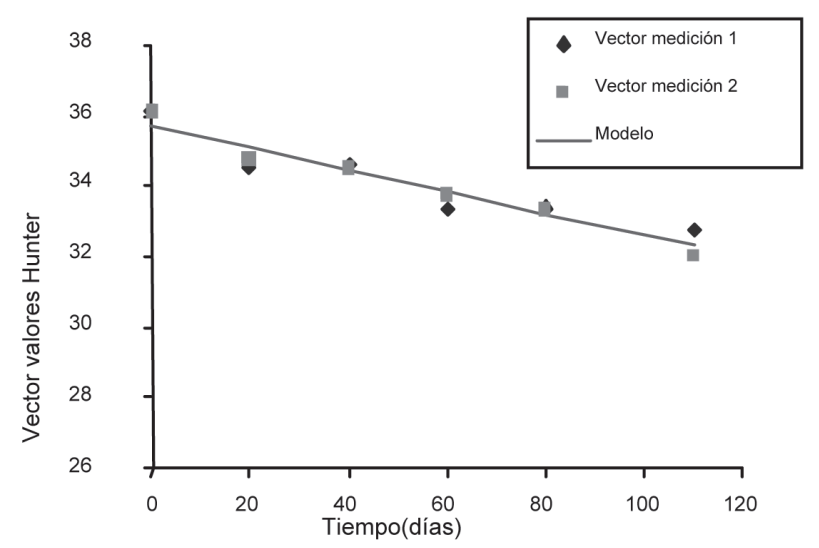

Figura 1. Vectores en función del tiempo para la temperatura de $40{ }^{\circ} \mathrm{C}$. Fuente: ( La autora y autores)

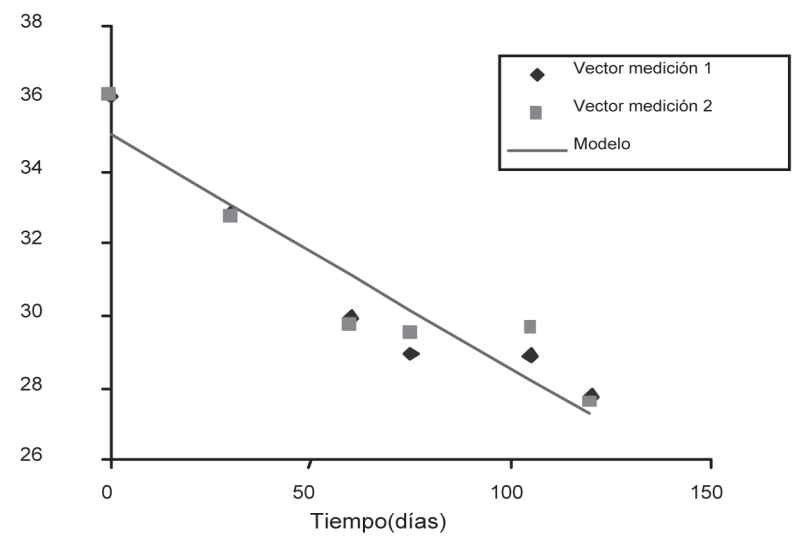

Figura 2. Vectores en función del tiempo para la temperatura de $45^{\circ} \mathrm{C}$.

Fuente: (La autora y autores)

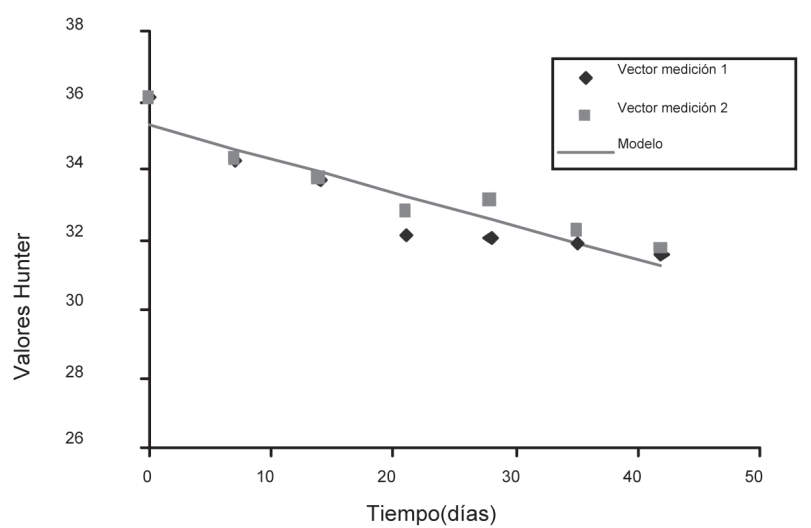

Figura 3. Vectores en función del tiempo para la temperatura de $50^{\circ} \mathrm{C}$. 
Cuadro 2. Equipos e instrumentos utilizados para determinar el color de la pasta de tomate.

Equipo
Fabricante/Modelo
Colorímetro
Cabeza
CR-310
datos
DP-301
Platos petri
P0mm x $15 \mathrm{~mm}$

Fuente: (La autora y autores)

Se puede observar en las tres figuras que a medida que pasa el tiempo el vector de color disminuye, es decir, el color rojo intenso de la pasta de tomate se va oscureciendo. Este deterioro presenta una tendencia lineal con pendiente negativa. Los datos fueron analizados estadísticamente y un análisis de residuos (no reportado) mostró que los datos se distribuyen aleatoriamente, es decir, no hay sesgo.

Las regresiones lineales obtenidas de estos gráficos se presentan a continuación:

$$
\begin{aligned}
& \text { Vector }=35,699-0,031 \cdot t \\
& \text { Vector }=34,989-0,064 \cdot t \\
& \text { Vector }=35,307-0,097 \cdot t
\end{aligned}
$$

Con las tres constantes obtenidas por mínimos cuadrados, y representadas por los valores de la pendiente en las ecuaciones (6), (7) y (8), para las tres temperaturas estudiadas se construyó un gráfico del 1 n $k$ en función de $1 / \mathrm{T}$.

La ecuación que se ajusta por mínimos cuadrados es:

$$
\ln k=33,33-11508 \cdot \frac{1}{T}
$$

El valor de energía de activación que se obtiene de esta ecuación es de $95680{ }^{\mathrm{J} /} \mathrm{mol}$.
Según estudios de paneles sensoriales llevados a cabo con jueces entrenados se obtuvieron valores de color Hunter Lab, para los cuales dichos jueces consideran que el producto ya no reúne las cualidades necesarias para el consumidor, cuando los valores son $\mathrm{L}=28,95$ , $a=+9,73$ y $b=+5,36$. El vector resultante de estos valores es 31,01 .

Al sustituir este resultado del vector en las ecuaciones (6), (7) y (8) se estima que la pasta de tomate incubada a $40^{\circ} \mathrm{C}, 45^{\circ} \mathrm{C}$ y $50^{\circ} \mathrm{C}$ tiene una vida útil de 150 días, 62 días y 44 días, respectivamente.

Se graficó el logaritmo de la vida útil a las temperaturas del estudio. La pseudotransformación resultante, ya que los ámbitos de temperatura son estrechos, se puede observar en la Figura 5 (Labuza, 1984).

Con la ecuación obtenida se puede estimar la vida útil de la pasta de tomate para diferentes temperaturas de almacenamiento.

$$
\text { Log vida útil }=4,259-0,053 \cdot T
$$

Y despejando la ecuación (10) se obtiene:

$$
\text { Vida útil }=10^{(4,259-0,053 \cdot T)}
$$

Donde $T$ está en ${ }^{\circ} \mathrm{C}$. 


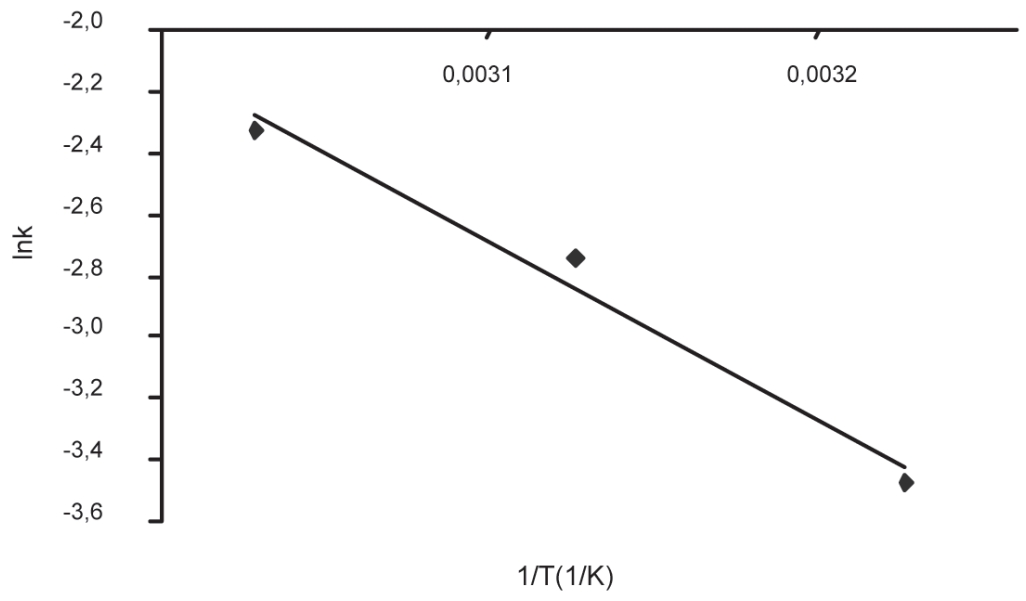

Figura 4. Gráfico del $1 n k$ en función de $1 / \mathrm{T}$.

Fuente: ( La autora y autores)

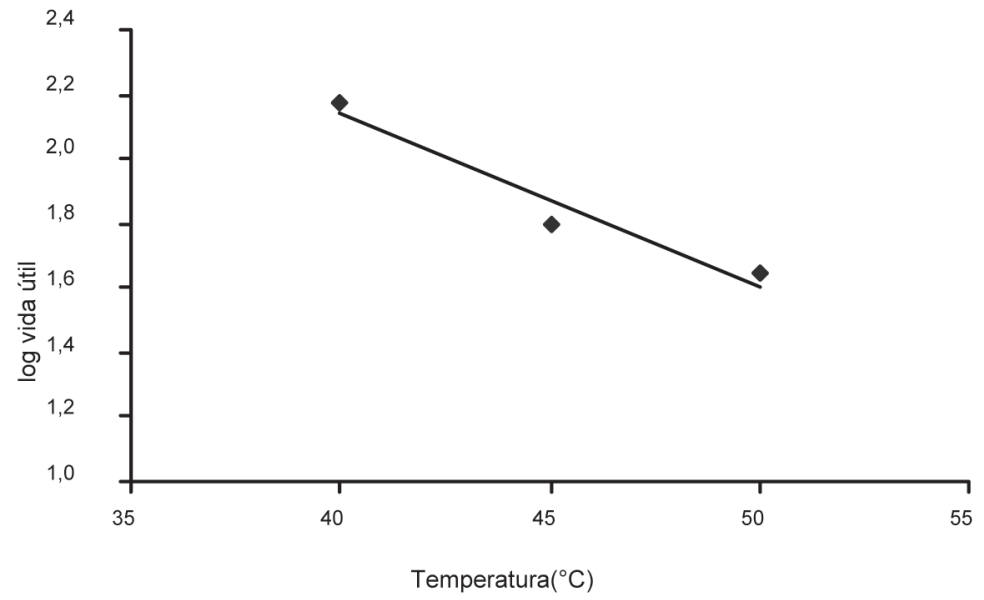

Figura 5. Gráfico del log vida útil en función de las temperaturas.

Fuente: ( La autora y autores) 


\section{CONCLUSIONES}

1. Se determinó que el deterioro del color de la pasta de tomate responde a un modelo de orden cero.

2. La energía de activación predicha mediante la ecuación de Arrhenius es de $96 \mathrm{KJ} / \mathrm{mol}$

3. La vida útil estimada del producto a $40^{\circ} \mathrm{C}$, $45^{\circ} \mathrm{C}$ y $50^{\circ} \mathrm{C}$ es de 150 días, 62 días y 44 días, respectivamente.

4. Con los tiempos y las temperaturas estudiadas se obtuvo una ecuación general para estimar la vida útil de este producto para diferentes temperaturas de almacenamiento; esta ecuación está descrita por: Vida útil =

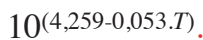

5. La ecuación de vida útil establecida permite predecir el comportamiento del producto a diferentes condiciones de temperatura que pueden encontrarse en las diferentes regiones del país.

\section{BIBLIOGRAFÍA}

Casp, A. y April, J.(1999). Procesos de conservación de alimentos. Madrid: Ediciones Mundi-Prensa.

Centro Nacional de Ciencia y Tecnología de Alimentos (CITA). (2005). Curso Teórico Práctico: Vida útil de alimentos aplicado a la industria. San José Costa Rica, (2005).

Díaz, J. (2004). Formulación de un producto colorante, a nivel de laboratorio, con una presentación líquida utilizando el extracto de la Bixa orellana(achiote) y evaluación de su estabilidad o vida útil de anaquel. Proyecto de graduación para optar al grado de Licenciatura en Ingeniería Química, Universidad de Costa Rica, San José, Costa Rica.

García, C. (2008). Estimación de la vida útil de un producto alimenticio pasteurizado y uno acidificado mediante pruebas aceleradas. Proyecto de graduación para optar al grado de Licenciatura en Ingeniería Química, Universidad de Costa Rica, San José, Costa Rica.

Kuntz, L. ( 19991). Accelerated Shelf Life Testing. Nueva York: Weeks Publishing Co.

Labuza, T.P. (1984). Aplication of chemical kinetics to deterioration of foods. Journal of Chemical Education 61(4): 348-358.

Labuza, T.P. \& Schmidt, M.K.(1985). Accelerated shelf-life dating of foods. Food Technology, 39(9): 57- 134.

Melgaard, M. (1999). Sensory Evaluation Tecniques. Florida: Edición CRC Press.

Neter, J. (1983). Applied Linear Regresión Models. Illinois: Richard D. Irwin, Inc.

Potter, N. (1978). La ciencia de los alimentos ( $2^{\mathrm{a}}$ ed.). México D.F: Edutex S.A.

Rondón.E., Pacheco.E. \& Ortega.F.(2004). Estimación de la vida útil de un análogo comercial de mayonesa utilizando el factor Q10.Facultad de Agronomía, Universidad Central de Venezuela. 4(21),68.83.

\section{SOBRE LOS AUTORES}

\section{Claudia García Baldezón}

Licenciada en Ingeniería Química de la Universidad de Costa Rica. Coordinadora Evaluación sensorial RID-CAM, Unilever de Centroámerica.

\section{Manuel Molina Córdoba}

Ingeniero Químico, Profesor Asociado de la Escuela de Ingeniería Química de la Universidad de Costa Rica.

Correo electrónico: MANUEL.MOLINA@ucr. ac.cr

\section{Gerardo Chacón Valle}

Ingeniero Químico, Profesor Asociado de la Escuela de Ingeniería Química de la Universidad de Costa Rica.

Correo electrónico: GERARDO.CHACON@ ucr.ac.cr 